\title{
Reliability and Validity of the Chen ADHD Scale (C-ADHDS)
}

This article was published in the following Dove Press journal:

Neuropsychiatric Disease and Treatment

\author{
Yi-Lung Chen $\mathbb{D}^{1,2}$ \\ Vincent Chin-Hung Chen ${ }^{3,4}$ \\ Michael Gossop $\mathbb{1 D}^{5}$ \\ 'Department of Healthcare \\ Administration, Asia University, Taichung, \\ Taiwan; ${ }^{2}$ Department of Psychology, Asia \\ University, Taichung, Taiwan; \\ ${ }^{3}$ Department of Psychiatry, Chang Gung \\ Medical Foundation, Chiayi Chang Gung \\ Memorial Hospital, Chiayi, Taiwan; \\ ${ }^{4}$ School of Medicine, Chang Gung \\ University, Taoyuan, Taiwan; ${ }^{5}$ National \\ Addiction Centre, Institute of Psychiatry, \\ King's College London, London, UK
}

Correspondence: Vincent Chin-Hung Chen Department of Psychiatry, Chang Gung Memorial Hospital, Chiayi Branch, Puzi, Chiayi, Taiwan

Email cch1966@gmail.com
Purpose: This study aimed to establish the Chen ADHD Scale and to examine its reliability and validity.

Patients and Methods: We recruited 114 individuals diagnosed with attentiondeficit/hyperactivity disorder (ADHD) and 39 non-ADHD controls aged between 5 and 18 years. Their parents completed the Chen ADHD Scale, Chinese versions of the SNAP-IV, and Strengths and Difficulties Questionnaire (SDQ). We examined the psychometric properties of Chen ADHD Scale, including test-retest reliability, internal consistency, construct validity, convergent and divergent validity.

Results: Receiver operating characteristic analysis was performed to calculate the area under the curve (AUC), sensitivity, and specificity of the Chen ADHD Scale for predicting ADHD. The Chen ADHD Scale demonstrated satisfactory test-retest reliability (intraclass correlation $=0.916)$, internal consistency (alpha $=0.966$ to 0.978$)$, a good model fit for a two-factor structure (inattention and hyperactivity-impulsivity) and good convergent and divergent validity with SNAP-IV and SDQ. The AUC of Chen ADHD Scale for predicting ADHD was 0.944. The optimal cut-off value of Chen ADHD Scale with impairment requirement for predicting ADHD was 37 with a sensitivity of 0.87 and a specificity of 0.97 . Conclusion: The Chen ADHD Scale is a reliable and valid instrument for screening ADHD symptoms in clinical settings in Taiwan.

Keywords: ADHD, scale, reliability, validity, psychometric

\section{Introduction}

Attention-deficit/hyperactivity disorder (ADHD) is one of the most common mental disorders in children with recent epidemiology studies indicating prevalence rates ranging from $6.7 \%$ to $10.1 \% .^{1,2}$ Attention deficit and hyperactivityimpulsivity are the two core features of symptoms that are noted in ADHD. ${ }^{3}$ It has been reported that ADHD contributes to a significant fraction of the global burden of disease in children, ${ }^{4}$ and economic burden on patients, families, and third-party payers. ${ }^{5}$ Given the growing awareness of ADHD, the importance of the assessment, diagnosis, and treatment of ADHD has been emphasized. ${ }^{6}$

Various tools have been developed to examine to evaluate ADHD in children and adolescents, and these measurements generally can be categorized into two types: clinical diagnosis-based approach and empirical-quantitative approach. ${ }^{7}$ Clinical diagnosis-based measurements are developed directly based on the diagnostic system, such as the Diagnostic and Statistical Manual of Mental Disorders (DSM) system, and these serve as a diagnostic assistance tool. Examples include the Connors Third Edition (Conners3), ${ }^{8}$ Swanson, Nolan, Pelham-IV Teacher and 
Parent Rating Scale (SNAP-IV), ${ }^{9}$ and Vanderbilt Assessment Scale. ${ }^{10}$ Among these tools, some of them (eg, Conners 3, SNAP-IV, Vanderbilt Assessment Scale) also evaluate ADHD-related behavioral, emotional problems, such as oppositional-defiant disorder, conduct disorder, anxiety, depression and academic performance.

Some researchers have used the empirical-quantitative approach to describe the psychopathology of ADHD instead of the diagnostic system. ${ }^{11}$ Assessment tools adopting this approach include Achenbach System of Empirically Based Assessment of Child Behavior Checklist (CBCL), ${ }^{12}$ Strengths and Weaknesses of ADHD Symptoms and Normal Behavior ${ }^{13}$ and Brown Attention-Deficit Disorder Scales. ${ }^{14}$ These scales are usually not strictly based on the criteria presented in the Diagnostic and Statistical Manual of Mental Disorders (DSM). ${ }^{13-15}$ Instead, their scale development is empirically derived through a series of quantitative analyses to determine the overlap of behavioral characteristics as a means of deriving specific dimensions of psychopathology. ${ }^{16}$ Some disadvantages of these tools are being considered. These tools are usually broadband scales, which generally focus on several psychopathologies (eg, emotional-behavioral problems) across many subscales, not specific to ADHD. Because of the lack of specificity, these broadband scales usually have few items for ADHD, as a result, the reliability in the ADHD subscales of broadband scales would be less optimal and unable to provide a robust measure of ADHD. ${ }^{17}$

Two possible limitations are considered in these aforementioned clinical diagnosis-based measurements. First, most of the assessments do not include the functional impairment results from ADHD symptoms. These measures focusing on symptoms tend to underestimate the importance of functional impairment, which is a crucial component in the diagnosis of mental disorders, ${ }^{18}$ like ADHD. Without an evaluation of functional impairment, the symptom severity of ADHD cannot be well gauged. The lack of screening items of functional impairment in the rating scale is also likely to overestimate the potential prevalence. ${ }^{19}$ Second, many assessments adopted a 4-point Likert scale. However, it has been reported that the Likert scale items are more vulnerable to bias from confounding factors and subject to the ceiling effect and more timeconsuming than visual analogue scale items. ${ }^{20}$

For rating scales, there is a special type of design, discretized analog scale, to improve measurement accuracy. This design allows the rater to use numeric, visual- spatial and verbal descriptive anchors to assess the domain simultaneously. ${ }^{21}$ In this study, we aimed to design an ADHD assessment tool based on the model of discretized analog scale and DSM-5 criteria including function impairment. We further comprehensively examined the reliability and validity by using a clinical sample of Taiwanese children and adolescents to test the feasibility of this scale to evaluate ADHD.

\section{Methods}

\section{Participants}

The sample consisted of 153 participants aged between 5 and 18. This included 114 individuals with ADHD from a teaching hospital and 39 non-ADHD controls. The study recruited ADHD and non-ADHD participants through a poster advertisement and outreach to outpatients in a medical center. All of the ADHD cases met the Diagnostic and Statistical Manual of Mental Disorders-5 diagnostic criteria for ADHD (American Psychiatric Association, 2013). These individuals with ADHD and non-ADHD were received a diagnostic interview by a psychiatrist to confirm their presence of ADHD diagnosis. All participants were invited for a re-test within one to two months. However, of them, $41.8 \%$ (64/153) of participants (49 individuals with ADHD with stable symptoms and similar medication dosage as well as 15 individuals with non-ADHD) accepted this invitation and participated in the followed up for a re-test at their scheduled return visit. All information for these children and adolescents with ADHD and without ADHD was reported by their parents. All data collected in this study is specifically for the development of the ADHD assessment tool, not for other research purposes. Written informed consent was obtained from all participating children and their parents. This study was conducted in accordance with the Declaration of Helsinki and approved by the Institutional Review Board of Chiayi Chang Gung Memorial Hospital (approval number: 201900694B0).

\section{The Chen ADHD Scale (C-ADHDS)}

The C-ADHDS is a 20-item rating scale developed to assess ADHD symptoms based on the DSM-5. The first 9 items and items 10 to 18 are corresponding to the symptom criteria for inattention and hyperactive-impulsive based on the DSM-5. The example items were "When speaking to $\mathrm{him} / \mathrm{her}$, it seems that he/she is not listening?" for inattention and "He/she often interrupts other people's conversations or activities" for hyperactive-impulsive, respectively. 
A 10-point visual analogue scale ranging through 0 (no), 1-3 (mild), 4-6 (moderate), 7-9 (marked), and 10 (extreme) is used to evaluate the severity of ADHD symptoms. The scale used numeric and verbal descriptive anchors simultaneously for a rating of severity of ADHD symptoms. This design has been used in other scales, such as the Sheehan disability scale. $^{21}$ The item 19 measures functional impairment in social, academic, or occupation with a score of 0 for "none," 1 for "mild," 2 for "moderate," and 3 for "severe." Item 20 is used to measure the quality of life with a visual analogue scale ranging from 0 (the worst) to 100 (the best). The C-ADHDS is designed for children and adolescents (7-18 years old) and available for parent-, selfand, teacher-report. The C-ADHDS assessed in this study was based on the parent's report.

\section{The Chinese Version of the Swanson, Nolan, and Pelham IV (SNAP-IV)}

The SNAP-IV is a 26-item scale developed by Swanson and colleagues to rate ADHD and oppositional defiant symptoms, designed for children age $6-17 .{ }^{22}$ The SNAPIV uses a 4-point Likert scale with a score of 0 for "not at all," 1 for "just a little," 2 for "quite a bit," and 3 for "very much". 9 A higher score in the SNAP-IV indicates more severe ADHD and oppositional defiant symptoms. The Chinese version of SNAP-IV and its norm are validated and developed by Gau and colleagues. High internal consistency and test-retest reliability were reported with a range of Cronbach's alpha of 0.88 to 0.90 and an intraclass correlation of 0.59 to $0.72 .^{23}$ We used the Chinese SNAP-IV based on parent-reported to compared to C-ADHDS

\section{The Chinese Version of the Strengths and Difficulties Questionnaires (SDQ)}

The SDQ is a 25-item behavioral screening questionnaire designed to assess a broader area of different psychological adjustment of children and adolescents. We used four subscales in the SDQ including emotional symptoms, conduct problems, hyperactivity/inattention, and peer relationship problems. A higher score in these subscales of SDQ indicates more severe behavioral emotional difficulties. High internal consistency was reported with a range of 0.40 to 0.86 between different subscales and rater forms. ${ }^{24}$ The SDQ assessed in this study was based on the parent's report.

\section{Statistical Analysis}

The descriptive results were shown and tested as frequency and percentage and Chi-Squared Test $\left(\chi^{2}\right)$ with
Yates's correction for categorical variables (ie, sex and the functional impairment measured in the C-ADHDS) and as mean and standard deviation and independent two sample $t$-test for continuous variables (ie, the total scores of subscales for SNAP-IV, C-ADHDS, and SDQ) between participants with 114 ADHD and 39 non-ADHD. A twosided $\mathrm{p}$ value $\leq 0.05$ was considered statistically significant in this study. Considering multiple comparisons in the SDQ subscales (4 subscales), the Bonferroni corrected $\mathrm{p}$-value was reported in the independent $t$-test analyses for the SDQ subscales.

Several indexes of reliability and validity for C-ADHDS were examined comprising internal consistent reliability of Cronbach's alpha, test-retest reliabilities of Pearson's $r$ and intraclass correlation (ICC) using two-way random effect model, consistency, for average measures, convergent and divergent validity, and sensitivity and specificity.

There were two parts to analyze the convergent and divergent validity of C-ADHDS. First, we conducted a confirmatory factor analysis (CFA) to determine factor validity. According to Dumenci and colleagues, ${ }^{25}$ there are three possible factor structures of ADHD in scale development: (a) a one-factor model of inattention and hyperactive-impulsive, (b) a correlated two-factor model of inattention (items 1-9) and hyperactive-impulsive (items 10-18), and (c) a hierarchical model of a general inattention and hyperactive-impulsive factor plus two specific factors of inattention and hyperactive-impulsive (See supplementary Figure 1). The Chi-square change test was used for model selection. It has been proposed that three to four items for one latent factor are preferable; $^{26}$ however, in the C-ADHDS, there were nine items for one latent factor. We, therefore, used the item parceling to represent each factor and item parceling has been used in scale validation. ${ }^{27,28}$

Specifically, the nine indicators for each factor were divided into three parcels based on items 1 to 3,4 to 6 , and 7 to 9 for inattention and items 10 to 12,13 to 15 , and 16 to 18 for hyperactivity-impulsivity. The averages of the three sets of indicators were used to create three parcel-level indicators for providing a more parsimonious measure of the inattention and hyperactivity-impulsivity factors. Model fit was assessed with multiple criteria: Chi-Squared Test $(\chi 2)$, Comparative Fit Index (CFI), Tucker-Lewis Index (TLI), and Root Mean Square Error of Approximation (RMSEA). Second, we further examined the convergent and divergent validity 
between the C-ADHDS and well-established measures (SNAP-IV and SDQ) using Pearson's r.

Sensitivity and specificity were examined, and four methods were used to classify ADHD based on the C-ADHDS. The first two methods used the continuous sum score C-ADHDS with or without impairment requirement to classify ADHD. Receiver operating characteristic (ROC) analysis was performed to calculate the area under the curve (AUC), sensitivity, and specificity of C-ADHDS for predicting ADHD. The optimal cut-off points for C-ADHDS were determined using the maximum value of the Youden index (sensitivity plus specificity minus 1). The other two methods were based on the symptom-count criterion from the full diagnostic criteria stated in the DSM-5 with or without impairment requirement, in which a score of 4 (moderate) or above was coded as the symptomatic presence of this behavior. The presence of an impairment requirement was based on the functional impairment in which a score of 1 (mild) or above. Therefore, the presence of a potential diagnosis of ADHD was defined as having a symptomatic response for at least six of the nine inattention items or six of the nine hyperactivity-impulsivity items with or without a functional impairment response from the C-ADHDS. In addition to the general ROC, we further conducted the covariate-adjusted receiver operating characteristic (AROC) to adjust for the confounding effects from demographics (ie, sex and age).

\section{Results}

\section{Sample Characteristics}

Among the 153 participants, the 114 individuals with ADHD were more likely to be boys, younger, have higher ADHD symptoms (based on the scores of SNAP-IV and the C-ADHDS), and behavioral emotional difficulties (based on the score of subscales of SDQ), compared to 39 individuals with non-ADHD, with Ps $<0.01$ (Table 1).

\section{Internal Consistent Reliability}

We found that the C-ADHDS has excellent internal consistency reliability with a Cronbach's alpha of 0.98 for whole symptoms items (item 1 to item 18), 0.97 for the subscale of inattention (item 1 to item 9) and 0.97 for the subscale of hyperactive-impulsive (item 10 to item 18), respectively.

\section{Test-Retest Reliability}

We found that C-ADHDS has excellent test-retest reliabilities with a Pearson's correlation of 0.85 and ICC of 0.92 , respectively. The mean duration of the follow-up period was 44.5 days for the re-retest.

\section{Confirmatory Factor Analysis}

The $\chi^{2}$ change test indicated that the hierarchical threefactor model fit significantly better than the one-factor model (ie, $\Delta \chi^{2}(6)=124.1 ; \mathrm{P}<0.001$ ), and the correlated two-factor model (ie, $\Delta \chi 2(5)=12.7 ; \mathrm{P}=0.027$ ). The results of confirmatory factor analysis for the C-ADHDS were satisfactory (ie, $\chi 2=6.42$, $\mathrm{df}=3, \mathrm{P}=0.092$, CFI $=$ 0.996, TLI $=0.984$, and RMSEA $=0.087)$. All standardized factor loadings were significant at $\mathrm{p}<0.001$ (Figure 1).

\section{Convergent and Divergent Validity}

These findings suggest that this model fitted well in the construct of the two ADHD symptom domains. For comparison between C-ADHDS and SNAP-IV and SDQ, the inattention and hyperactive-impulsive of C-ADHDS had stronger correlations with corresponding ADHD symptoms in SNAP-IV and the subscale of hyperactivity of SDQ. By contrast, relatively weak associations were found between C-ADHDS and the other subscales of SDQ (Table 2).

\section{Sensitivity and Specificity}

ROC with the sum of continuous C-ADHDS and symptom-count criterion based on the DSM-5 was used to classify ADHD and to calculate sensitivity and specificity. First, the results of ROC analysis for the prediction ability of C-ADHDS for ADHD are shown in Figure 2, which yielded an AUC of 0.944. Furthermore, after controlling for demographics (ie, sex and age) using AROC, a high adjusted AUC of 0.935 was still observed.

Based on the maximum value of the Youden index, we observed an optimal cut-off value for C-ADHDS without impairment requirement $t$, which was equal to or greater than 37 , with a sensitivity of 0.88 and a specificity of 0.90 were found (Table 3). Furthermore, when we considered the impairment requirement by classifying individuals with ADHD when they reported impairment in ADHD symptoms and scored C-ADHDS equal to or greater than 37, a sensitivity of 0.87 and a specificity of 0.97 for C-ADHDS were observed. On the other hand, a more conservative result of the number of ADHD was found when using the symptom-count criterion based on the DSM-5, the sensitivity and specificity were 0.70 and 0.95 
Table I Demographics, Chen ADHD Scale, Swanson, Nolan, and Pelham IV and Strengths and Difficulties Questionnaires in Our Sample

\begin{tabular}{|c|c|c|c|}
\hline Variables & ADHD $n=1 / 4$ & Non-ADHD n=39 & Statistics \\
\hline \multicolumn{4}{|l|}{ Sex, N (\%) } \\
\hline Boys & $95(83.3)$ & $22(56.4)$ & $<0.001$ \\
\hline Girls & $19(16.7)$ & $17(43.6)$ & \\
\hline Age, Mean (SD) & $10.5(2.9)$ & $13.2(4.7)$ & 0.003 \\
\hline \multicolumn{4}{|c|}{ Chen ADHD Scale, Mean (SD) } \\
\hline Inattention & 44.9 (19.9) & $11.0(11.9)$ & $<0.001$ \\
\hline Hyperactivity & $36.7(21.2)$ & $5.2(7.2)$ & $<0.001$ \\
\hline Quality of life & $66.5(21.0)$ & $83.1(14.9)$ & $<0.001$ \\
\hline \multicolumn{4}{|l|}{ Impairment, N (\%) } \\
\hline None & $5(4.4)$ & $34(87.2)$ & $<0.001$ \\
\hline Mild & $38(33.3)$ & $5(12.8)$ & \\
\hline Moderate & $52(45.6)$ & $0(0.0)$ & \\
\hline Severe & $19(16.7)$ & $0(0.0)$ & \\
\hline \multicolumn{4}{|c|}{ Swanson, Nolan, and Pelham IV, Mean (SD) } \\
\hline Inattention & $14.5(5.3)$ & $4.5(3.9)$ & $<0.001$ \\
\hline Hyperactivity & $12.0(6.2)$ & $2.1(2.2)$ & $<0.001$ \\
\hline \multicolumn{4}{|c|}{ Strengths and Difficulties Questionnaires, Mean (SD) } \\
\hline Conduct problems & $3.5(1.8)$ & $1.2(1.2)$ & $<0.001$ \\
\hline Hyperactivity & $6.6(2.2)$ & $\mathrm{I} .5(\mathrm{I} .7)$ & $<0.001$ \\
\hline Emotional symptoms & $3.4(2.3)$ & $1.4(1.6)$ & $<0.001$ \\
\hline Peer problems & $3.5(2.0)$ & $1.7(1.8)$ & $<0.001$ \\
\hline
\end{tabular}

Note: Bonferroni corrected p-value was applied for Strengths and Difficulties Questionnaires.

for without impairment requirement and 0.69 and 0.97 for with impairment requirement, respectively (Table 3).

\section{Discussion}

In this study, our finding suggested that C-ADHDS is a reliable and valid instrument for diagnosing child and adolescent ADHD disorders for clinical and research use. The psychometric characteristics showed that the C-ADHDS developed in this study was not only an updated ADHD assessment tool to fit the DSM- 5 criteria but also an improvement in the scale performance by using visual analogue scale items to obtain satisfactory psychometric properties.

Adequate internal consistency reliability and test-retest reliability are essential for the application of selfadministrated scales and the present study showed excellent internal consistency reliability with Cronbach's alpha of 0.97 to 0.98 , and test-retest index with Pearson's $r$ correlation of 0.85 and ICC of 0.92 for the total C-ADHDS score. In these respects, the scale is comparable with or even better than other common ADHD assessment tools. For consistency reliability (ie, Cronbach's alpha), the C-ADHDS was comparable with IOWA Conners' rating scale $(0.78$ to 0.91$),{ }^{29}$ ADHD rating scale $(0.86)^{30}$ and Chinese version of SNAP-IV (0.88 to 0.90$).{ }^{23}$ Similarly, test-retest reliability in most several tools of ADHD assessment ranged from 0.6 to 1.0, including the subscale of hyperactivity-inattention of strengths and difficulties questionnaire with Pearson's $r$ correlations of 0.60 of $0.82,{ }^{31}$ and the subscale of attention problems of the Chinese version of CBCL with Pearson's $r$ correlations of 0.66 of $0.87,{ }^{32}$ the Chinese version of SNAP-IV of with Pearson's $r$ correlations of 0.68 to 0.73 and ICCs of 0.67 to $0.72,{ }^{23}$ IOWA Conners' rating scale with Pearson's $\mathrm{r}$ correlations of 0.73 to $0.80,{ }^{29}$ ADHD rating scale with Pearson's $r$ correlations of $0.76,{ }^{30}$ and Vanderbilt ADHD diagnostic parent rating scale with Pearson's $r$ correlations of 0.75 to $0.95 .{ }^{10}$ Generally, the ADHD specific assessment tools had better reliability than broadband scales possibly because of more items in ADHD specific assessment than in broadband scales. ${ }^{17}$ 


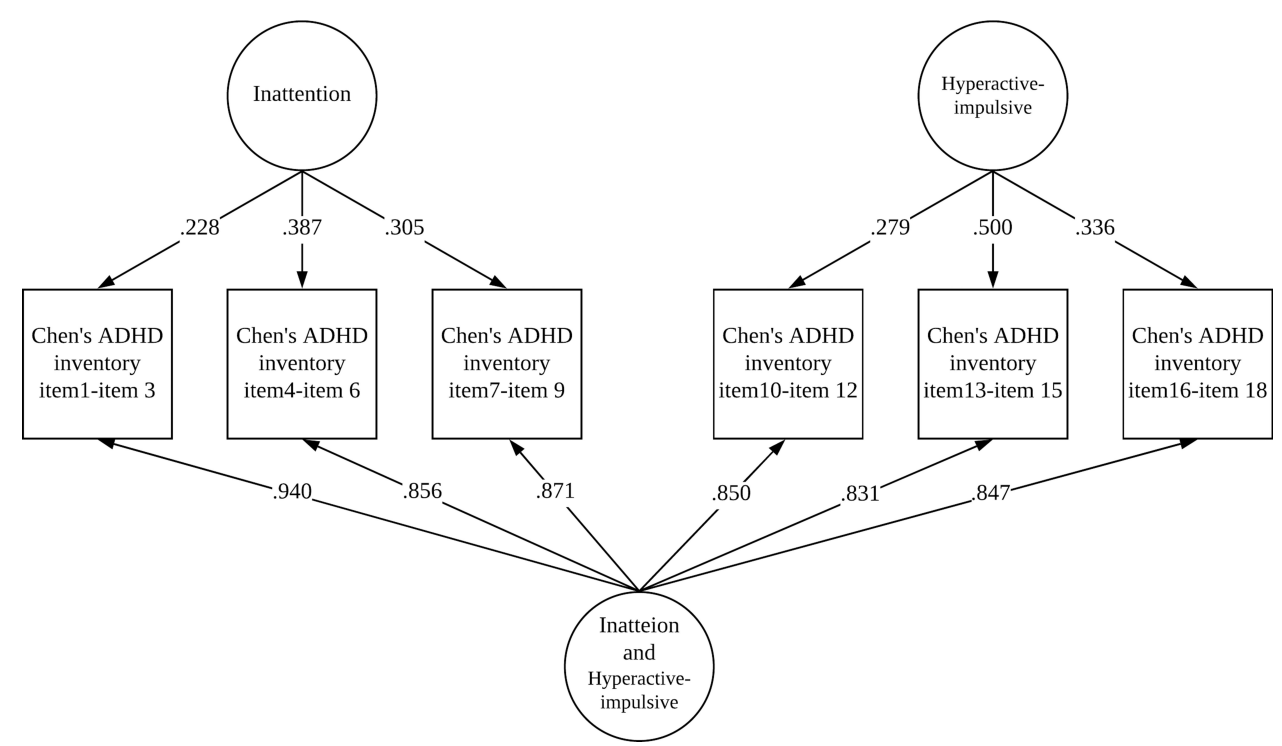

Figure I The confirmatory factor analysis for the Chen ADHD Scale and its standardized factor loading. The confirmatory factor analysis for the C-ADHDS was satisfactory $(\chi 2=6.42, \mathrm{df}=3, \mathrm{P}=0.092, \mathrm{CFI}=0.996, \mathrm{TLI}=0.984$, and RMSEA $=0.087)$.

Good construct validity and convergent and divergent validity of the C-ADHDS was supported by the model fit index of the CFA and the associations between C-ADHDS and SNAP-IV and SDQ. The hierarchical three-factor model of inattention and hyperactivity-impulsivity in the C-ADHDS has the best model fit than the one-factor model and the correlated two-factor model. This finding was supported by the current literature, ${ }^{25,33}$ indicating that ADHD is not a unitary construct and inattention is related to hyperactivity-impulsivity, and also distinct with hyperactivity-impulsivity after the consideration of the general factor of inattention and hyperactivity-impulsivity. This result supports the classification of subtypes of ADHD in the DSM (ie, predominantly Inattentive type,

Table 2 Correlations Between Chen ADHD Scale and Other Clinical Questionnaires

\begin{tabular}{|l|l|l|}
\hline \multirow{2}{*}{ Clinical Questionnaire } & \multicolumn{2}{|l|}{ Chen ADHD Scale } \\
\cline { 2 - 3 } & Inattention & Hyperactivity-impulsivity \\
\hline Swanson, Nolan, and Pelham & & \\
IV & & \\
Inattention & 0.88 & 0.75 \\
Hyperactivity-impulsivity & 0.75 & 0.91 \\
\hline Strengths and Difficulties & & \\
Questionnaires & & \\
Conduct problems & 0.61 & 0.60 \\
Hyperactivity & 0.77 & 0.78 \\
Emotional symptoms & 0.48 & 0.41 \\
Peer problems & 0.42 & 0.36 \\
\hline
\end{tabular}

Note: All analyses reached statistical significance with $P<0.00$. predominantly Hyperactive/Impulsive type, and Combined type). ${ }^{3,25}$ In addition to constructs within the C-ADHDS, these two scales of C-ADHDS also have good convergent and divergent validity corresponding to two well-known measures, SNAP-IV of ADHD symptoms and SDQ of emotional-behavioral problems. Despite the high correlations between the subscales of hyperactivity of SDQ and C-ADHDS (Pearson's $r$ correlations of 0.770 to 0.779), there remain moderate associations between emotional-behavioral problems (ie, conduct problems, emotional symptoms and peer problems) and C-ADHDS with

ROC Curve for Chen ADHD Scale Area Under the Curve $=0.944$

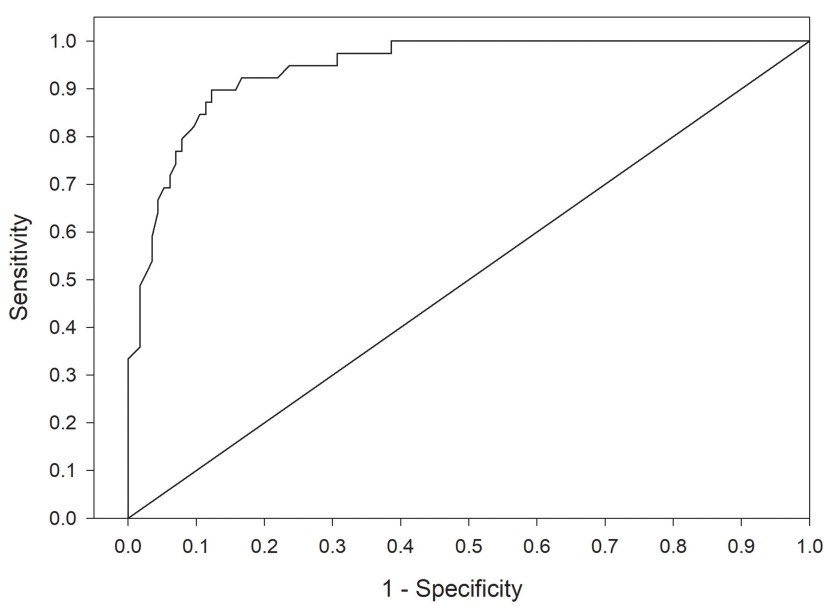

Figure 2 Receiver operating characteristic curve analysis predicting ADHD using the Chen ADHD Scale. 
Table 3 Sensitivity and Specificity of Chen ADHD Scale Based on Sum Score and Symptom-Count Criterion with or without Impairment of the DSM-5

\begin{tabular}{|l|l|l|l|l|l|l|l|l|}
\hline \multirow{2}{*}{} & \multicolumn{3}{|l|}{ Chen ADHD Scale } \\
\cline { 2 - 8 } & \multicolumn{2}{l|}{$\begin{array}{l}\text { Optimal Cut-Off Value } \\
\text { without Impairment } \\
\text { Requirement }\end{array}$} & \multicolumn{2}{l|}{$\begin{array}{l}\text { Optimal Cut-Off Value } \\
\text { with Impairment } \\
\text { Requirement }\end{array}$} & $\begin{array}{l}\text { Without Impairment } \\
\text { Requirement } \\
\text { (Based on the DSM-5) }\end{array}$ & $\begin{array}{l}\text { With Impairment } \\
\text { Requirement } \\
\text { (Based on the DSM-5) }\end{array}$ \\
\hline $\begin{array}{l}\text { Tool } \\
\text { diagnosis }\end{array}$ & Positive & Negative & Positive & Negative & Positive & Negative & Positive & Negative \\
\hline Positive & 100 & 4 & 99 & $\mathrm{I}$ & 80 & 2 & 79 & I \\
\hline Negative & 14 & 35 & 15 & 38 & 34 & 37 & 35 & 38 \\
\hline & $\begin{array}{l}\text { Sensitivity } \\
=0.88\end{array}$ & $\begin{array}{l}\text { Specificity } \\
=0.90\end{array}$ & $\begin{array}{l}\text { Sensitivity } \\
=0.87\end{array}$ & $\begin{array}{l}\text { Specificity } \\
=0.97\end{array}$ & $\begin{array}{l}\text { Sensitivity } \\
=0.70\end{array}$ & $\begin{array}{l}\text { Specificity } \\
=0.95\end{array}$ & $\begin{array}{l}\text { Sensitivity } \\
=0.69\end{array}$ & $\begin{array}{l}\text { Specificity } \\
=0.97\end{array}$ \\
\hline
\end{tabular}

Abbreviation: DSM, Diagnostic and Statistical Manual of Mental Disorders.

Pearson's $r$ correlations of 0.35 to 0.61 . However, these constructs in these subscales from SDQ were considered common comorbidities with ADHD, and some ADHD assessments also include items measuring these comorbidities, such as oppositional defiant disorder symptoms in Conners Rating Scales-Revised, SNAP-IV, and Vanderbilt Assessment Scale and anxiety and depression in Vanderbilt Assessment Scale. ${ }^{17}$ For this reason, individuals with high ADHD tendency may also to some extent manifest emotional-behavioral problems, and consequently tend to score higher on these subscales from SDQ.

Our study demonstrated that the C-ADHDS has an excellent predictive ability to identify potential ADHD based on the AUR of 0.94 and the high sensitivity and specificity in two different classification approaches (the optimal cut-off value of 37 and the symptom-count criterion based on the DSM-5). For the method of optimal cutoff value with impairment requirement, it achieved an optimal balance between sensitivity and specificity with a sensitivity of 0.87 and a specificity of 0.97 . We observed that the impairment requirement plays an important role in differentiating clinical and non-clinical participants with ADHD, which was reflected in the change of specificity from 0.90 (without impairment requirement) to 0.97 (with impairment requirement). Unlike the cut-off value method, the result from the symptom-count criterion based on the DSM-5 has an extremely high specificity of 0.97 , with a cost of reasonable sensitivity of 0.69 . With these psychometric properties, if researchers consider the false positive as important as false negative in the screening of ADHD, the cut-off value approach would be the appropriate method. By contrast, if the medical cost is high for false positive and researchers want to avoid false positive, the symptom-count criterion approach may be preferred over the cut-off value approach. For example, in a community with a high stigma of mental disorder, a highly sensitive assessment tool may lead to a higher proportion of falsepositive results and cause possible over-responses. In this case, an assessment tool with extremely high specificity would be more preferable. In addition, the symptom-count criterion based on the DSM-5 without and with the requirement of functioning impairment both had similar sensitivity ( 0.70 for without vs 0.69 for with requirement) and specificity ( 0.95 for without vs 0.97 for with requirement). A possible reason may be that we included clinical patients with ADHD and most of them had significant functional impairment from ADHD symptoms. This explanation was supported by our results wherein $95.6 \%$ of individuals with ADHD reported meeting the requirement of functional impairment. However, it has been demonstrated that the utility of functioning impairment is a basis of the assessment of mental disorders in the community setting. $^{34}$

\section{Limitation}

Several limitations of this study should be mentioned. Our results were obtained from a small, non-representative clinical sample in Taiwan and the generalizability is limited to other populations. There were significant differences in terms of demographics (ie, sex and age) between the ADHD and non-ADHD groups. However, two possible reasons suggest such differences may have 
little impact on our results. First, the convergent and divergent validity are estimated using the within-group comparison, not the between-group comparison. Thus, between-group differences have no impact on convergent and divergent validity. Second, we used AROC to adjust the possible confounding of demographics, and we observed comparably high AUC and adjusted AUC values (ie, 0.944 and 0.935 , respectively). This result indicated that demographics may have limited confounding effects in our reliability and validation examination. Finally, this was a parent-report study and the reliability and validity data for the child and adolescent self-report and teacher report were still lacking. Hence, the normative data for the general population and the validation study for self-report and teacher-report are warranted for further studies.

\section{Conclusions}

The C-ADHDS is a reliable and valid instrument to be applied in clinical practice and research to assess ADHD based on the latest DSM-5. Our results demonstrated that the C-ADHDS has good to excellent internal consistency reliability, test-retest reliability, construct validity, convergent and divergent validity of the C-ADHDS for emotional and behavioral problems (SDQ) and ADHD symptoms (SNAP-IV) and sensitivity and specificity of clinical diagnosis of ADHD.

\section{Data Sharing Statement}

The data that support the findings of this study are available from the corresponding author upon reasonable request.

\section{Funding}

There is no funding to report.

\section{Disclosure}

The authors have no conflicts of interest to declare for this work.

\section{References}

1. Chen YL, Chen WJ, Lin KC, et al. Prevalence of DSM-5 mental disorders in a nationally representative sample of children in Taiwan: methodology and main findings. Epidemiol Psychiatr Sci. 2020;29 (e15):1-9. doi:10.1017/S2045796018000793

2. Thomas R, Sanders S, Doust J, et al. Prevalence of attention-deficit/ hyperactivity disorder: a systematic review and meta-analysis. Pediatrics. 2015;135(4):e994-1001. doi:10.1542/peds.2014-3482

3. American Psychiatric Association. Diagnostic and Statistical Manual of Mental Disorders. Fifth Edition. (DSM-5). Washington, DC: American Psychiatric Pub; 2013
4. Erskine HE, Ferrari AJ, Polanczyk GV, et al. The global burden of conduct disorder and attention-deficit/hyperactivity disorder in 2010. J Child Psychol Psychiatry. 2014;55(4):328-336. doi:10.1111/jcpp.12186

5. Matza LS, Paramore C, Prasad M. A review of the economic burden of ADHD. Cost Eff Resour Alloc. 2005;3(1):5. doi:10.1186/14787547-3-5

6. Froehlich TE, Lanphear BP, Epstein JN, et al. Prevalence, recognition, and treatment of attention-deficit/hyperactivity disorder in a national sample of US children. Arch Pediatr Adolesc Med. 2007;161(9):857-864. doi:10.1001/archpedi.161.9.857

7. Krol NP, De Bruyn EE, Coolen JC, et al. From CBCL to DSM: a comparison of two methods to screen for DSM-IV diagnoses using CBCL data. J Clin Child Adolesc Psychol. 2006;35(1):127-135. doi:10.1207/s15374424jccp3501_11

8. Conners CK. Conners. third. (Conners 3). Los Angeles, CA: Western Psychological Services; 2008.

9. Swanson JM, Kraemer HC, Hinshaw SP, et al. Clinical relevance of the primary findings of the MTA: success rates based on severity of ADHD and ODD symptoms at the end of treatment. J Am Acad Child Adolesc Psychiatry. 2001;40(2):168-179. doi:10.1097/00004583200102000-00011

10. Bard DE, Wolraich ML, Neas B, et al. The psychometric properties of the Vanderbilt attention-deficit hyperactivity disorder diagnostic parent rating scale in a community population. J Dev Behav Pediatr. 2013;34(2):72-82. doi:10.1097/DBP.0b013e31827a3a22

11. de Wolff MS, Vogels AGC, Reijneveld SA. The empirical versus DSM-oriented approach of the child behavior checklist. European J Psychol Assessment. 2014;30(1):22-30. doi:10.1027/1015-5759/ a000164

12. Achenbach TM, Rescorla L. Manual for the ASEBA School-Age Forms \& Profiles: An Integrated System of Multi-Informant Assessment. Burlington, VT:: Aseba; 2001.

13. Brites C, Salgado-Azoni CA, Ferreira TL, et al. Development and applications of the SWAN rating scale for assessment of attention deficit hyperactivity disorder: a literature review. Braz J Med Biol Res. 2015;48(11):965-972. doi:10.1590/1414-431×20154528

14. McKee ML, Mortimer JE, Maricle DE, et al. Brown attention-deficit disorder scales. In: Goldstein S, Naglieri JA, editors. Encyclopedia of Child Behavior and Development. Boston, MA: Springer US; 2011:302-303.

15. Achenbach TM, Edelbrock CS. Manual for the Child: Behavior Checklist and Revised Child Behavior Profile. Burlington: University of Vermont, Department of Psychiatry; 1983.

16. Allison Bender H, Auciello D, Morrison CE, et al. Comparing the convergent validity and clinical utility of the behavior assessment system for children-parent rating scales and child behavior checklist in children with epilepsy. Epilepsy Behav. 2008;13(1):237-242. doi:10.1016/j.yebeh.2008.03.007

17. Collett BR, Ohan JL, Myers KM. Ten-year review of rating scales. V: scales assessing attention-deficit/hyperactivity disorder. $J$ Am Acad Child Adolesc Psychiatry. 2003;42(9):1015-1037. doi:10.1097/01. CHI.0000070245.24125.B6

18. Leu S-H, Chou J-Y, Lee P-C, et al. Validity and reliability of the Chinese version of the Sheehan Disability Scale (SDS-C). AsiaPacific Psychiatry. 2015;7(2):215-222. doi:10.1111/appy.12182

19. Bolton P, Wilk CM, Ndogoni L. Assessment of depression prevalence in rural Uganda using symptom and function criteria. Soc Psychiatry Psychiatr Epidemiol. 2004;39(6):442-447. doi:10.1007/s00127-0040763-3

20. Voutilainen A, Pitkaaho T, Kvist T, et al. How to ask about patient satisfaction? The visual analogue scale is less vulnerable to confounding factors and ceiling effect than a symmetric Likert scale. $J$ Adv Nurs. 2016;72(4):946-957. doi:10.1111/jan.12875

21. Sheehan DV, Harnett-Sheehan K, Raj BA. The measurement of disability. Int Clin Psychopharmacol. 1996;11(Suppl 3):89-95. doi:10.1097/00004850-199606003-00015 
22. Swanson JM, Schuck S, Porter MM, et al. Categorical and dimensional definitions and evaluations of symptoms of ADHD: history of the SNAP and the SWAN rating scales. Int $J$ Edu Psychol Assessment. 2012;10(1):51-70.

23. Gau SS, Shang CY, Liu SK, et al. Psychometric properties of the Chinese version of the Swanson, Nolan, and Pelham, version IV scale - parent form. Int J Methods Psychiatr Res. 2008;17(1):35-44. doi:10.1002/mpr.237

24. Liu SK, Chien YL, Shang CY, et al. Psychometric properties of the Chinese version of strength and difficulties questionnaire. Compr Psychiatry. 2013;54(6):720-730. doi:10.1016/j.comppsych.2013.01.002

25. Dumenci L, McConaughy SH, Achenbach TM. A hierarchical threefactor model of inattention-hyperactivity-impulsivity derived from the attention problems syndrome of the teacher's report form. School Psych Rev. 2004;33(2):287-301. doi:10.1080/0279601 5.2004.12086249

26. Bollen KA. Structural Equations with Latent Variables. New York: John Wiley; 1989.

27. Osman A, Breitenstein JL, Barrios FX, et al. The fear of pain questionnaire-III: further reliability and validity with nonclinical samples. $J$ Behav Med. 2002;25(2):155-173. doi:10.1023/ A:1014884704974

28. Verdugo Alonso MÁ, Arias Martínez B, Gómez Sánchez LE, et al. Development of an objective instrument to assess quality of life in social services: reliability and validity in Spain. Int J Clin Health Psychol. 2010.
29. Waschbusch DA, Willoughby MT. Parent and teacher ratings on the IOWA conners rating scale. J Psychopathol Behav Assess. 2008;30 (3):180. doi:10.1007/s10862-007-9064-y

30. Faries DE, Yalcin I, Harder D, et al. Validation of the ADHD rating scale as a clirlician administered and scored instrument. J Atten Disord. 2001;5(2):107-115. doi:10.1177/108705470100500204

31. Goodman R. Psychometric properties of the strengths and difficulties questionnaire. J Am Acad Child Adolesc Psychiatry. 2001;40 (11):1337-1345. doi:10.1097/00004583-200111000-00015

32. Leung PW, Kwong S, Tang C, et al. Test-retest reliability and criterion validity of the Chinese version of CBCL, TRF, and YSR. J Child Psychol Psychiatry. 2006;47(9):970-973. doi:10.1111/j.14697610.2005.01570.x

33. Toplak ME, Sorge GB, Flora DB, et al. The hierarchical factor model of ADHD: invariant across age and national groupings? J Child Psychol Psychiatry. 2012;53(3):292-303. doi:10.1111/j.1469-7610.2011.02500.x

34. Yang HJ, Soong WT, Kuo PH, et al. Using the CES-D in a two-phase survey for depressive disorders among nonreferred adolescents in Taipei: a stratum-specific likelihood ratio analysis. J Affect Disord. 2004;82(3):419-430. doi:10.1016/j.jad.2004.04.008
Neuropsychiatric Disease and Treatment

\section{Publish your work in this journal}

Neuropsychiatric Disease and Treatment is an international, peerreviewed journal of clinical therapeutics and pharmacology focusing on concise rapid reporting of clinical or pre-clinical studies on a range of neuropsychiatric and neurological disorders. This journal is indexed on PubMed Central, the 'PsycINFO' database and CAS, and
Dovepress

is the official journal of The International Neuropsychiatric Association (INA). The manuscript management system is completely online and includes a very quick and fair peer-review system, which is all easy to use. Visit http://www.dovepress.com/testimonials.php to read real quotes from published authors. 